\title{
Reflets
}

Revue ontaroise d'intervention sociale et communautaire

\section{Boivin, Louise et Mark Fortier, L'économie sociale, l'avenir d'une illusion, Montrál, Fides, 1998.}

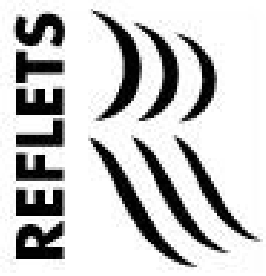

\section{Denyse Côté}

Volume 5, numéro 1, printemps 1999

Pratiques et développement économique communautaire

URI : https://id.erudit.org/iderudit/026260ar

DOI : https://doi.org/10.7202/026260ar

Aller au sommaire du numéro

Éditeur(s)

Reflets : Revue ontaroise d'intervention sociale et communautaire

ISSN

1203-4576 (imprimé)

1712-8498 (numérique)

Découvrir la revue

Citer ce compte rendu

Côté, D. (1999). Compte rendu de [Boivin, Louise et Mark Fortier, L'économie sociale, l'avenir d'une illusion, Montrál, Fides, 1998.] Reflets, 5(1), 192-197. https://doi.org/10.7202/026260ar

Tous droits réservés (C) Reflets : Revue ontaroise d'intervention sociale et communautaire, 1999
Ce document est protégé par la loi sur le droit d'auteur. L'utilisation des services d'Érudit (y compris la reproduction) est assujettie à sa politique d'utilisation que vous pouvez consulter en ligne.

https://apropos.erudit.org/fr/usagers/politique-dutilisation/ 


\section{L'économie sociale, l'avenir d'une illusion...}

BOIVIN, Louise et Mark FORTIER, Montrál, Fides, 1998.

par Denyse Côté

Professeure, Département de travail social, Université du Québec à Hull

L'illusion dont nous font part les auteurs de ce livre n'est pas en réalité celle de l'économie sociale, mais plutôt celle du projet de société lié à l'économie sociale qui a fait couler beaucoup d'encre au Québec depuis le début des années 1990 et auquel s'est associé le gouvernement Bouchard depuis 1996. Ces auteurs réagissent en effet fortement à l'engouement qui a traversé les milieux communautaires et syndicaux depuis le Sommet économique de l'économie et de l'emploi. Phénomène récurrent et compréhensible, cet engouement ne peut d'aucune façon, selon eux, remplacer l'analyse des solutions proposées à la situation actuelle de l'emploi et de l'économie ou servir de placebo en cette époque de crise de l'emploi.

Avant de présenter ce livre, il s'avère essentiel de situer le contexte politique québécois. Contrairement au gouvernement Harris en Ontario, le gouvernement Bouchard a développé au Québec des façons pour le moins originales d'implanter les politiques néolibérales. On ne parle pas ici de copier l'Alberta ou l'Ontario, mais bien de développer une nouvelle solidarité sociale autour de la création d'emplois, du déficit zéro et du désinvestissement de l'État. L'économie sociale est centrale à cette stratégie. Là où le bât blesse, selon Boivin et Fortier, c'est que les guerres sont confondues, l'économie sociale traduite en panacée ou en solution unique devient dangereuse pour la démocratie et pour la société québécoise. 
Soulignons pour la lectrice ou le lecteur ontarien ou peu familier avec la conjoncture québécoise qu'il s'avère nécessaire ici de différencier clairement le développement économique communautaire du projet de société que constitue maintenant l'économie sociale au Québec. À ceux qui trouveront ces distinctions spécieuses, nous répondrons qu'elles sont au contraire incontournables. Car si on peut identifier l'économie sociale à un champ d'action et d'intervention, le volume de Boivin et Fortier démontre par contre hors de tout doute que s'y profile aussi une réalité d'une toute autre nature: celle de l'économie sociale comme stratégie de sortie de crise et comme fondement d'un nouveau contrat social au Québec.

Nous ne pouvons qu'appuyer certaines prémisses partagées par les auteurs: l'émergence de l'économie sociale comme solution unique a dernièrement remplacé au Québec le débat de société rendu nécessaire par la crise du travail. La mise en place des mesures d'encouragement à l'économie sociale a également permis la progression de la logique marchande qui s'est retrouvée élargie à de nouvelles sphères, celle des services dits de proximité par exemple. Ceci n'a pas permis par contre d'en réduire la précarisation ou la dévalorisation. Le nouveau contrat social proposé, car c'est bien de ceci dont il s'agit, définit encore, ou plutôt renforce aujourd'hui la définition de la citoyenneté par le biais de l'exercice du travail salarié. Or, limiter la définition de la citoyenneté, ou faire passer obligatoirement la définition de la citoyenneté responsable par le biais du travail salarié, associer la cohésion sociale ou pis encore associer les liens sociaux constitutifs de l'identité personnelle et communautaire au travail salarié, s'avère une stratégie dangereuse en cette fin de siècle : il faudrait plutôt tenter de sortir du paradigme du travail salarié puisque celui-ci traverse une crise sans précédent.

«L'intérêt de l'économie sociale repose sur ses capacités à réinvestir le travail salarié d'une nouvelle aura, voire d'une moralité: l'utilité sociale. En tentant de transformer l'ensemble des activités bénévoles mais aussi militantes en autant d'emplois sous le vocable d'économie sociale, on semble vouloir redonner un sens au travail en l'investissant de la mission de réparer la cohésion sociale que l'on dit par ailleurs mise à mal». (p. 226) 
C'est pourquoi nous recommandons fortement ce livre à toute personne s'intéressant de près à l'évolution de la conjoncture québécoise, en particulier à sa mouvance communautaire. Il s'agit essentiellement d'un recueil d'essais critiques à l'égard des nouveaux discours sur l'économie sociale, d'un livre qui nous sort de la morosité de cette pensée unique qui pèse malheureusement lourdement sur le milieu communautaire. Il se situe de plein-pied dans la tradition critique et propose un recul analytique à facettes multiples. Si par contre ce sont les dernières expériences en matière d'économie sociale ou de développement économique communautaire qui vous intéressent, vous risquez d'être déçu. Nous résumerons brièvement dans les lignes qui suivent les analyses présentées.

Paul Grell expose dans son article comment l'économie sociale constitue une sortie de crise à visage humain issue du partenariat entre les syndicats, le secteur communautaire, le secteur public (le gouvernement) et le patronat. Elle permet un financement complémentaire des services communautaires et des services publics. L'économie hybride qui en résultera se base sur «des compromis possibles de part et d'autre» (p. 195). Or ce langage récemment importé de l'économie sociale/solidaire et non encore digéré réagit mal selon lui aux questions simples, dont la suivante : pourquoi les gisements d'emplois que ferait émerger l'économie sociale n'ont-ils pas déjà été découverts par l'économie de marché? C'est qu'il s'agit selon lui des «services de réparation» dont parlait Erving Goffamn il y a déjà longtemps et qui, s'ils sont en expansion présentement, ne constituent pas moins des «situations intermédiaires» entre le travail et l'assistance. De plus, le discours sur les services de proximité et sur l'économie sociale devient plus normatif qu'analytique, ce qui en fait un nouveau credo plutôt qu'une solution.

Michel Pazarelli et Gilles Tardif soutiennent par ailleurs que les perspectives démocratiques de l'économie sociale doivent être analysées dans le contexte actuel de dualisation socio-économique. Ils mettent en doute l'efficacité du modèle de l'économie sociale "qui prétend sauver les exclus par la réforme d'un pacte social censé faciliter à nouveau, par la création d'emplois, l'accès aux 
biens de consommation» (p. 59). Ce modèle de sortie de crise a été d'après eux imposé aux mouvements ouvrier, populaire et communautaire, à telle enseigne que "ces acteurs se retrouvent aujourd'hui déchirés par le sentiment de perdre le fruit de plusieurs années de lutte...» (p. 58). Si l'expression est forte, c'est que la mise en marche de l'économie sociale n'est pas, dans sa forme présente, une pratique émancipatrice puisque les acteurs n'ont pas été invités à en définir collectivement les balises (p. 60). Dans sa forme actuelle, l'économie sociale correspondrait plutôt à une pratique "communautique» associée à un modèle social où les règles du jeu sont préétablies, les finalités et les stratégies déterminées à l'avance par d'autres acteurs que ceux impliqués dans l'action communautaire. Ceci n'aura comme conséquence que d'amplifier le sentiment de dépossession "par la sollicitation de leur servitude volontaire aux programmes de l'État» (p. 67). L'économie sociale serait aussi dans sa forme actuelle un «objet de foi» dont les médiations et configurations pratiques ne garantissent aucunement un élargissement des processus démocratiques et une prise en charge de leur destin par les individus et les collectivités.

L'État québécois utilise l'économie sociale, selon Louise Boivin, pour mettre en place ses politiques néolibérales, en particulier celles reliées au travail obligatoire. Les actions gouvernementales prennent donc appui sur les discours portés par les promoteurs de l'économie sociale autour de la restructuration du rôle de l'État et de la solidarité sociale et ce, malgré l'absence au Québec de mesures obligatoires d'intégration en emploi («workfare»). Elle compare donc les mesures mises en oeuvre par le gouvernement québécois à celles suggérées par l'OCDE; elle s'arrête ainsi par exemple à la connotation négative maintenant donnée aux mesures «passives» de soutien au revenu, au renversement du fardeau que représentent les mesures dites d' "employabilité» (c'est la personne sans emploi qui doit maintenant s'assurer de son employabilité plutôt que le secteur privé ou public de l'offre d'emplois). Bref, tout ceci constitue pour elle un terrain fertile pour l'introduction du travail obligatoire («workfare»). 
Georges Lebel semble partager ce point de vue puisque pour lui l'économie sociale est une stratégie qui s'intègre parfaitement à la nouvelle idéologie néolibérale du "good governance». Celle-ci justifie la déconstruction de l'État régulateur qui, désormais, s'occupera plutôt de s'assurer que la nouvelle citoyenneté responsable s'adapte aux impératifs du marché. On assiste ainsi à un transfert de l'État vers le secteur associatif et communautaire et au développement correspondant d'un discours localiste qui fait le jeu du néolibéralisme. L'exigence de flexibilisation qui émane des politiques néolibérales se retrouve donc «à la source des propositions de reconnaissance de l'économie sociale» (p. 106). L'auteur examine aussi les transformations proposées ou déjà actualisées du cadre législatif qui, selon lui, systématisent le processus de dévolution et de privatisation (p. 123). Il souligne les dangers inhérents à la systématisation et à la bureaucratisation de la solidarité, de même que le fait que les services issus du secteur communautaire devront maintenant être responsables de l'application des normes nationales dans leurs domaines respectifs.

Enfin, selon Lucie Lamarche, les entreprises d'économie sociale seront le lieu privilégié de marchandisation des fonctions sociales que l'État déleste à une vitesse foudroyante (p.139). On déplace donc le «lieu d'arbitrage du social vers les collectivités en faisant de ces dernières le lieu de démonstration du besoin d'une citoyenneté plus fonctionnelle, c'est-à-dire plus responsable» (p. 142). On ne peut donc séparer la mise en place de mesures d'économie sociale de la désétatisation et de la dénationalisation. Différenciant avec raison les entreprises d'économie sociale du projet de l'économie sociale, l'auteure démontre comment ce projet ouvre la porte à la mise en place du caractère transactionnel des droits de la personne et du droit du travail.

Ce débat autour des finalités et des retombés de l'économie sociale s'est tissé sur la toile de fond de la situation des femmes et des revendications féministes de l'heure. Les féministes et les groupes de femmes y ont été très actives et très présentes sur le terrain: par le biais de la Marche des femmes de 1995, elles ont même permis (quoique peut-être dans une certaine mesure involontairement) à l'économie sociale d'émerger comme scénario 
macropolitique. Elles restent cependant dans l'ensemble partagées face à l'économie sociale et conscientes des doublestranchants de cette solution. En un mot, l'économie sociale ne correspond pas pour elles à la revendication des infrastructures sociales mise de l'avant lors de la Marche de 1995, et elles ont développé une critique et une pratique alternative en matière d'économie sociale. Diane Lamoureux présente dans son article cette analyse critique des mesures d'économie sociale du point de vue des femmes. La trousse proposée par Relais-femmes collige une série d'outils déjà rodés dans le cadre d'une formation offerte en 1997-98 aux quatre coins du Québec. On y retrouve la plate-forme féministe adoptée en décembre 1997 en matière d'économie sociale. On y retrouve également des textes et outils pédagogiques qui remettent en contexte les productions économiques marchandes et nonmarchandes ainsi qu'une explication de diverses stratégies conçues pour combattre l'appauvrissement des femmes. On s'y attarde plus particulièrement à la mise en place de projets locaux d'économie sociale et de développement communautaire qui conserveraient une visée alternative, une analyse critique et une volonté d'équité.

Pour obtenir cette trousse, contacter Relais-femmes au 110 Ste-Thérèse, bureau 301, Montréal H2Y 1 E6. 